\title{
290 Radical neck dissection
}

A Consists of removal of all the lymph-bearing structures between the midline, mandible, anterior border of sternomastoid and clavicle.

B The sternomastoid muscle, internal jugular vein, and submandibular gland are excised with the specimen.

C The upper end of the internal jugular vein is divided first.

D The accessory nerve is preserved.

E A chylous leak during a left-sided operation is recognized as milky fluid collecting in the lower part of the wound.

\section{Functional neck dissection}

A Aims to remove only nodes and tissue likely to be involved by tumour.

B Published indications include the clinically NO neck with high risk of occult metastases.

C Papillary carcinoma of the thyroid is a generally accepted indication.

D The accessory nerve is preserved.

E Crile never performed such a procedure.

\section{Pharyngeal pouch (Zenker's diverticulum)}

A Origin is between middle and inferior constrictor muscles (Dehiscence of Killian).

B The cause is traction by contracting tuberculous scar tissue.

C Associated symptoms include dysphagia and dyspnoea.

D Dohlman's operation is an endoscopic excision of the pouch.

$\mathrm{E}$ Inversion of the pouch is an alternative to excision. 